\title{
Software Package for Determining the Capillary Adsorption Potential in Irrigated Lands
}

\author{
R.A. Danielyan \\ Armenian National Agrarian University \\ razmikdanielyan@mail.ru
}

\section{A R T I C L E I N F O}

Keywords:

capillary adsorption potential, irrigation,

model,

moisture transfer equation, software package

\begin{abstract}
A B S T R A C T
In the current work, an analytical formula for determining the capillary adsorption potential (CAP) has been derived as a result of solving the moisture transfer equation. A universal software package program has been written in the Python language. The code enables to compute the values of CAP at any soil depth and at any time of irrigation for various soil parameters. To illustrate the code, the results of some numerical calculations are presented at the end of the work.
\end{abstract}

\section{Introduction}

Forecasting of capillary adsorption potential (CAP) in irrigated lands plays a specific role in hydromelioration. Optimal management of the water regime in irrigated lands ensures increase in crop yield capacity and improvement in fertility. CAP depends on various physicochemical parameters and mechanical characteristics of the soil. Therefore, the creation of a software package for predicting CAP in various types of lands with different soil parameters is of vital significance (Yeghiazaryan, 2006).

In the current work, the analytical formula for determining the CAP was obtained as a result of solving model equation of moisture transfer in the aeration zone. A universal software package is suggested to identify values of CAP for arbitrary soil parameters. The software package is written in Python language (https://ru.wikipedia.org/wiki/Python), which enables to compute values of CAP at any soil depth and at any time during the period of irrigation interval for different values of soil parameters. By using the code, it is possible to depict the dependence of the CAP on the depth of the soil, as well as its surface as a function of $\varphi(x, t)$ with two variables: the depth of the soil and the period of irrigation interval.

\section{Materials and methods}

The distribution of CAP via depth of soil and time of irrigation is described by the following moisture transfer equation (Tikhonov and Samarsky, 1977):

$$
\frac{\partial \varphi}{\partial t}=a^{2} \frac{\partial^{2} \varphi}{\partial^{2} x}-a_{1}
$$


Here $\varphi(x, t)$ is the required CAP:

$$
a^{2}=\frac{K_{0}}{\theta_{0}}, \quad a_{1}=\frac{e_{0}}{\theta_{0}},
$$

where $K_{0}$ - is the mean value of soil moisture transfer coefficient, $\theta_{0}$ - is the average value of differential moisture capacity, $e_{0}$ - is the absorption intensity of moisture by plant roots.

The equation (1) is supplemented with the boundary and initial conditions:

$$
\begin{gathered}
\left.\frac{\partial \varphi}{\partial x}\right|_{x=0}=\left.\bar{q}_{1} \frac{\partial \varphi}{\partial x}\right|_{x=l}=\bar{q}_{2}, \\
\varphi(x, 0)=q(x) .
\end{gathered}
$$

Here $\bar{q}_{1}$ and $\bar{q}_{2}$ are average velocities of moisture conductivity above and below ground surfaces respectively, $q(x)$ is the capillary adsorption potential when $t=0$. It leads to the following representation:

$$
q(x)=\alpha e^{-\beta x},
$$

where $\alpha$ and $\beta$ are known numerical parameters, which are determined experimentally. It should be noted that experimental data are well approximated with the representation (5).

Making similar reasoning as when constructing a solution to the classical initial-boundary value problem for the moisture transfer equation, one can make it sure that the solution to problems (1), (3), (4) admits the following form:

$$
\begin{aligned}
& \varphi(x, t)=\frac{\left(\bar{q}_{2}-\bar{q}_{1}\right) x^{2}}{2 l}+\bar{q}_{1} x+\frac{C_{0}}{2}+ \\
& +\sum_{n=1}^{\infty} C_{n} \cos \frac{\pi n}{l} x e^{-\left(\frac{a \pi n}{l}\right)^{2} t}+W_{0} t,
\end{aligned}
$$

where Fourier coefficients $C_{0}$ and $C_{n}$ are equal to:

$$
\begin{gathered}
C_{0}=\frac{2}{l} \int_{0}^{l} \psi(x) d x, \\
C_{n}=\frac{2}{l} \int_{0}^{l} \psi(x) \cos \frac{\pi n}{l} x d x,
\end{gathered}
$$

where

$$
\begin{gathered}
\psi(x)=q(x)-\frac{\left(\bar{q}_{2}-\bar{q}_{1}\right) x^{2}}{2 l}-q_{1} x, \\
W_{0}=\frac{2 a^{2}\left(\bar{q}_{2}-\bar{q}_{1}\right)}{l}-2 a_{1}=2\left(\frac{a^{2}\left(\bar{q}_{2}-\bar{q}_{1}\right)}{l}-a_{1}\right) .
\end{gathered}
$$

Placing (10) in (8) and (9) with the consideration of (5), and using the values of the following integrals:

$\int_{0}^{l} e^{-\beta x} \cos \frac{\pi n}{l} x d x= \begin{cases}\frac{\beta l^{2}\left(1+e^{-\beta l}\right)}{\pi^{2} n^{2}+\beta^{2} l^{2}}, & \text { if } n=2 k+1, \\ \frac{\beta l^{2}\left(1-e^{-\beta l}\right)}{\pi^{2} n^{2}+\beta^{2} l^{2}}, & \text { if } n=2 k .\end{cases}$

$\int_{0}^{l} x^{2} \cos \frac{\pi n}{l} x d x=\left\{\begin{array}{ll}-\frac{2 l^{3}}{\pi^{2} n^{2}}, & \text { if } n=2 k+1, \\ \frac{2 l^{3}}{\pi^{2} n^{2}}, & \text { if } n=2 k .\end{array} \quad n \neq 0\right.$

$\int_{0}^{l} x \cos \frac{\pi n}{l} x d x= \begin{cases}-\frac{2 l^{2}}{\pi^{2} n^{2}}, & \text { if } n=2 k+1, \\ 0, & \text { if } n=2 k .\end{cases}$

we get the following for the Fourier coefficients:

$C_{0}= \begin{cases}\frac{2 a}{\beta l}\left(1-e^{-\beta l}\right)-\frac{l}{3}\left(\bar{q}_{2}+2 \bar{q}_{1}\right), & \text { if } n=0, \beta \neq 0, \\ 2 a-\frac{l}{3}\left(\bar{q}_{2}+2 \bar{q}_{1}\right) & \text { if } n=0, \beta=0 .\end{cases}$

$C_{n}= \begin{cases}\frac{-2 l\left(\bar{q}_{2}-\bar{q}_{1}\right)}{\pi^{2} n^{2}}+\frac{2 \beta l a\left(1-e^{-\beta l}\right)}{\pi^{2} n^{2}+\beta^{2} l^{2}}, & \text { if } n=2 k+1 \\ \frac{2 l\left(\bar{q}_{2}+\bar{q}_{1}\right)}{\pi^{2} n^{2}}+\frac{2 \beta l a\left(1+e^{-\beta l}\right)}{\pi^{2} n^{2}+\beta^{2} l^{2}}, & \text { if } n=2 k .\end{cases}$

\section{Results and discussions}

First of all, we should specify the ranges of variation for the following basic parameters depending on the soil type:

1. Soil depth, $0 \leq x \leq l$ (м)

2. Period of irrigation interval $0 \leq t \leq T$ (day) 
3. Average velocity of water exchange on the soil surface $q_{1}$ (m/day)

4. Average velocity of water exchange under the soil surface $\bar{q}_{2}$ (m/day)

5. Parameters of CAP at the initial time period $a(\mathrm{~m})$ and $\beta(1 / \mathrm{m}) t=0$

6. Parameter application intervals $a^{2}\left(m^{2} / d a y\right)$ and $a_{1}$ (m/day)

Now we will shortly describe the code implementation. Let's enter starting parameters:

$$
l, t, \alpha, \beta, \bar{q}_{1}, \bar{q}_{2} a_{1}, a^{2} .
$$

Then the Python code computes coefficients of Fourier $C_{0}$ and $C_{n}$ in accordance with the formula (11) and (12). As a final step the code computes the values of capillary adsorption potential (CAP) $\varphi(x, t)$, designs surface $\varphi(x, t)$ as a function with two variables, as well as the CAP dependence on depth of soil at different period of irrigation intervals is computed by using the formula (6).

Below, the results for some numerical calculations are introduced.

Figure 1.1, 1.2 and Table 1 correspond to the following values of the main parameters:

$$
\begin{gathered}
l=1 \mathrm{~m}, T=2 \text { day, } \bar{q}_{1}=-1 \mathrm{~m} / \text { day, } \bar{q}_{2}=-0.5 \mathrm{~m} / \text { day, } \\
a_{1}=0.3 \mathrm{~m} / \text { day, } a^{2}=0.25 \mathrm{~m}^{2} / \text { day }, \\
\alpha=4 \mathrm{~m}, \beta=1 \mathrm{l} / \mathrm{m} .
\end{gathered}
$$

Table 1. The value of the capillary adsorption potential $\varphi(x, t)$ at various values of $x$ and $t^{*}$

\begin{tabular}{|c|c|c|c|}
\hline $\boldsymbol{N}$ & $\boldsymbol{x}$ & $\boldsymbol{t}$ & $\boldsymbol{\varphi ( x , \boldsymbol { 0 } )}$ \\
\hline 1 & 0.0 & 0.0 & 3.9993921772309675 \\
\hline 2 & 0.1 & 0.2 & 3.2479608008492193 \\
\hline 3 & 0.2 & 0.4 & 2.94178222304284 \\
\hline 4 & 0.3 & 0.6 & 2.757037162850827 \\
\hline 5 & 0.4 & 0.8 & 2.6573340811101973 \\
\hline 6 & 0.5 & 1.0 & 2.6159797040328328 \\
\hline 7 & 0.6 & 1.2 & 2.6139960682039005 \\
\hline 8 & 0.7 & 1.4 & 2.63916068818068 \\
\hline 10 & 0.8 & 1.6 & 2.684347466635085 \\
\hline 11 & 1 & 2.0 & 2.821970456452652 \\
\hline
\end{tabular}

*Composed by the author.

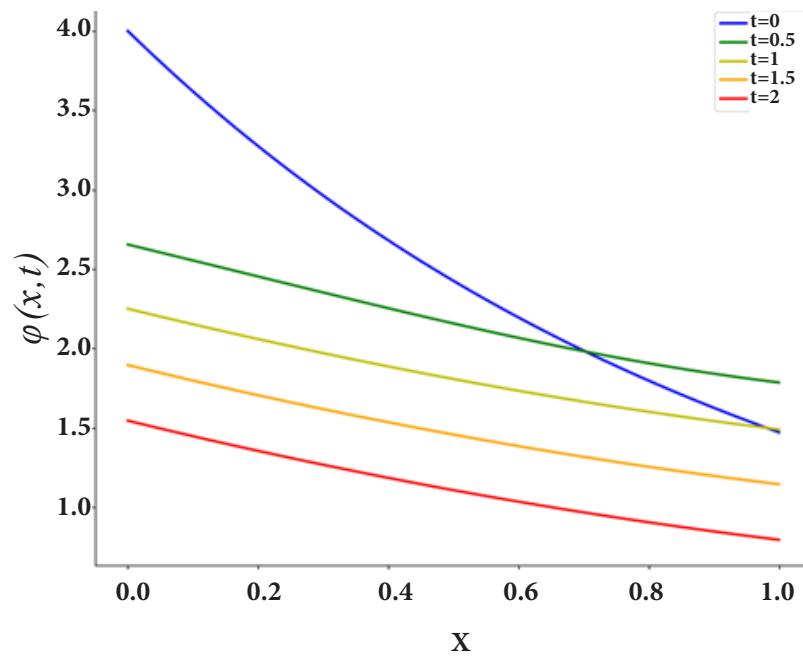

Figure 1.1. Dependence of the capillary adsorption potential on the soil depth at different values of $t$ (composed by the author).

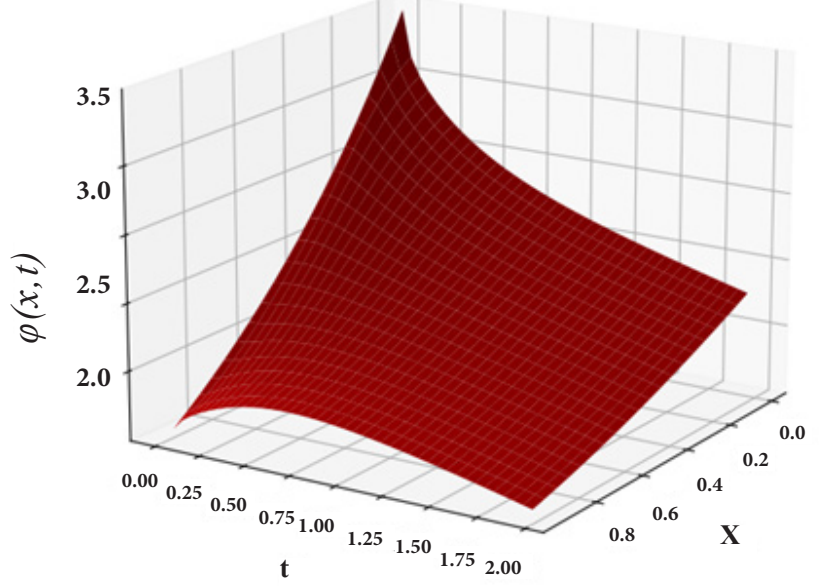

Figure 1.2. Two-dimensional distribution of the capillary adsorption potential (composed by the author).

Figure 2.1, 2.2 and Table 2 correspond to the following values of the main parameters:

$$
\begin{gathered}
l=0.1 \mathrm{~m}, T=2 \text { day, } \\
\bar{q}_{1}=-0.5 \mathrm{~m} / \text { day, } \bar{q}_{2}=-0.5 \mathrm{~m} / \text { day, } \\
a_{1}=0.3 \mathrm{~m} / \text { day, } a^{2}=0.25 \mathrm{~m}^{2} / \text { day, } \\
\alpha=4 \mathrm{~m}, \beta=0 \mathrm{l} / \mathrm{m} .
\end{gathered}
$$


Table 2. The value of the capillary adsorption potential $\varphi(x, t)$ at various values of $x$ and $t^{*}$

\begin{tabular}{|c|c|c|c|}
\hline $\mathbf{N}$ & $\boldsymbol{x}$ & $\boldsymbol{t}$ & $\boldsymbol{\varphi ( x , 0 )}$ \\
\hline 1 & 0.0 & 0.0 & 4.000010132114987 \\
\hline 2 & 0.1 & 2.0 & 2.7750000000000004 \\
\hline
\end{tabular}

*Composed by the author.

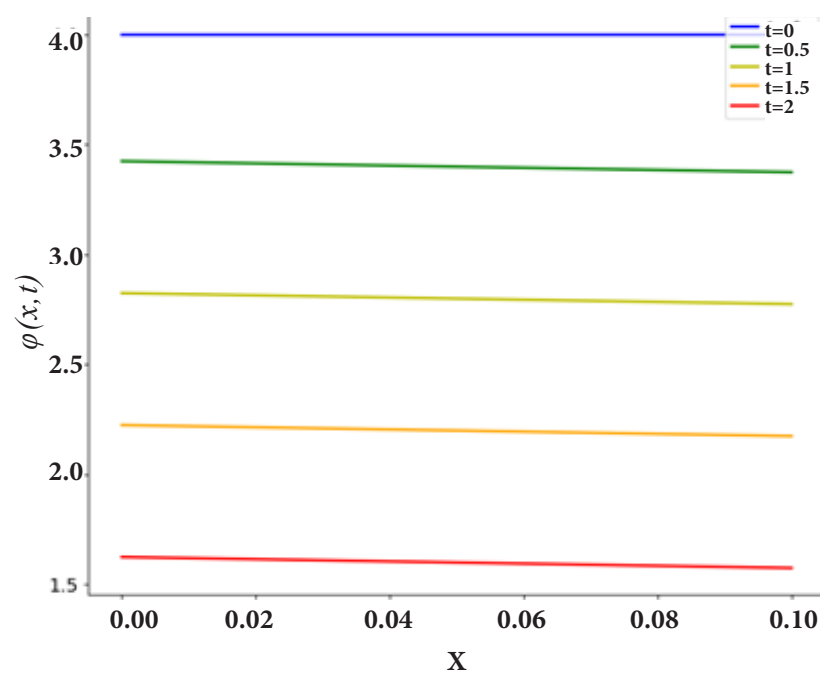

Figure 2.1. Dependence of the capillary adsorption potential on the soil depth at different values of $t$ (composed by the author).

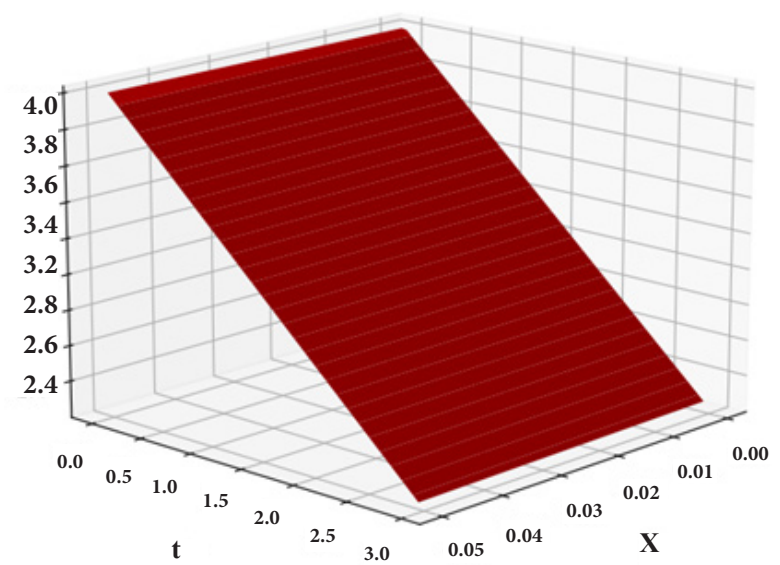

Figure 2.2. Two-dimensional distribution of the capillary adsorption potential (composed by the author).
Figure 3.1, 3.2 and Table 3 correspond to the following values of the main parameters:

$$
\begin{gathered}
l=0.5 \mathrm{~m}, T=2 \text { day, } \\
\bar{q}_{1}=-0.75 \mathrm{~m} / \text { day, } \bar{q}_{2}=-0.5 \mathrm{~m} / \text { day, } \\
a_{1}=0.3 \mathrm{~m} / \text { day, } a^{2}=0.25 \mathrm{~m}^{2} / \mathrm{day}, \\
\alpha=4 \mathrm{~m}, \beta=0.5 \mathrm{l} / \mathrm{m} .
\end{gathered}
$$

Table 3. The value of the capillary adsorption potential $\varphi(x, t)$ at various values of $x$ and $t^{*}$

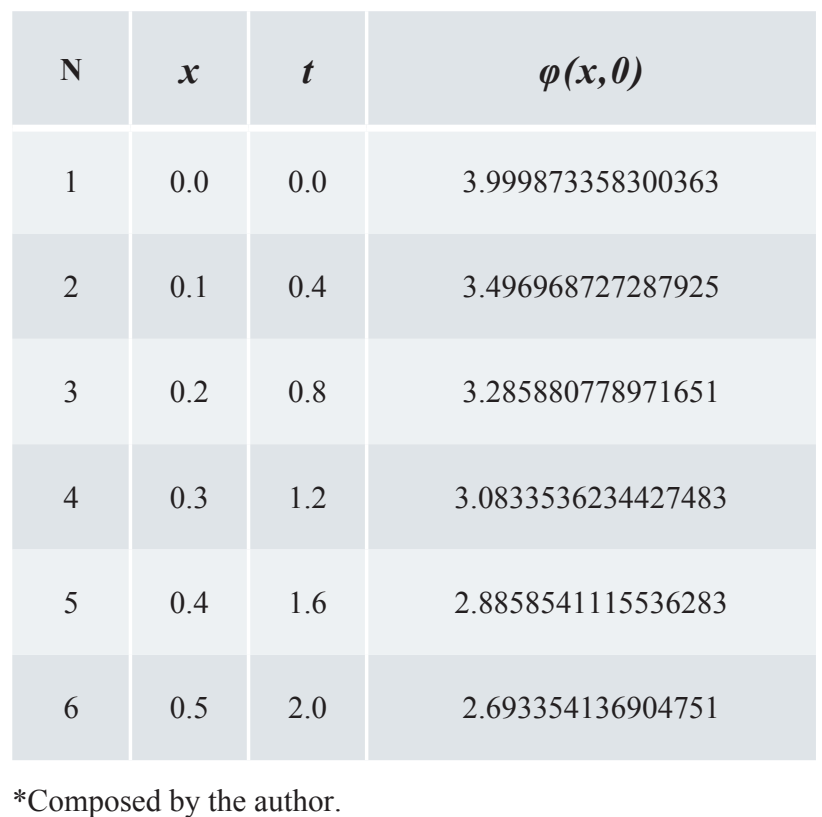

*Composed by the author.

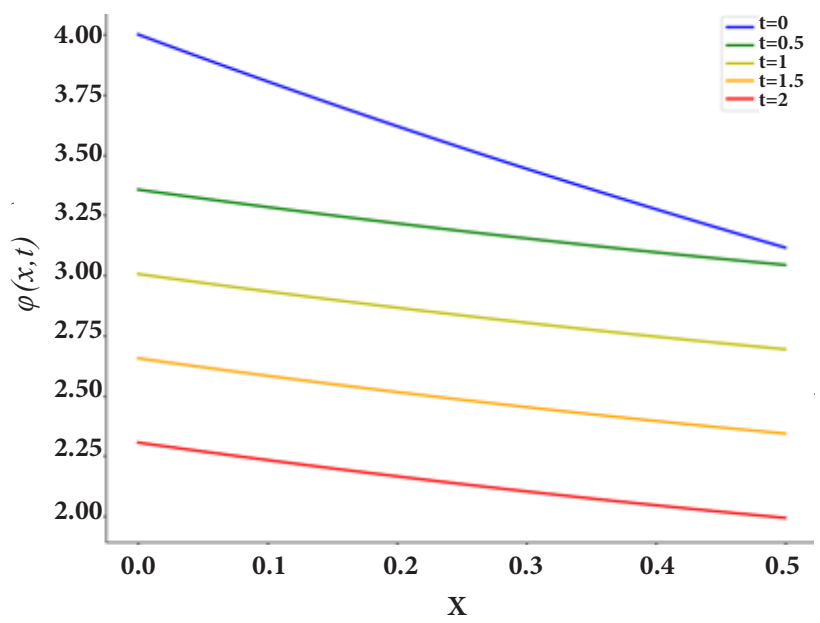

Figure 3.1. Dependence of the capillary adsorption potential on the soil depth at different values of $t$ (composed by the author). 


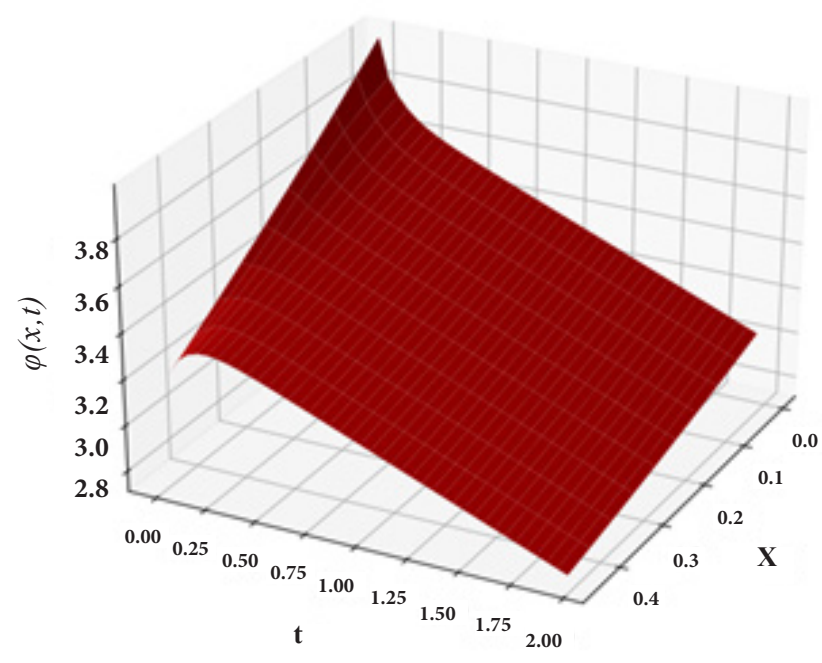

Figure 3.2. Two-dimensional distribution of the capillary adsorption potential (composed by the author).

\section{Conclusion}

An analytical-numerical method for determining the capillary adsorption potential (CAP) in irrigated lands is recommended in the current work. Software package program written in Python language has been designed. The code enables to estimate CAP depending on depth of soil and period of irrigation for different parameters of the soil. After entering the initial parameters the code computes the values of CAP as well as depicts two dimensional distributions of CAP.

\section{References}

1. Python Programming Language, https://ru.wikipedia. org/wiki/Python (accessed in May, 2021).

2. Tikhonov, A.N., Samarsky, A.A. (1997). Equations of Mathematical Physics, Moscow, Nauka, - 735 p.

3. Yeghiazaryan, G.M. (2006). Prediction and Regulation of Water-Salt Regime in Irrigated Lands, Yerevan, ANAU, - 133 p. 